\title{
Design of Base Flow for Streamline-Traced Hypersonic Inlet
}

\author{
Lianjie Yue ${ }^{1}$, Yabin Xiao ${ }^{2}$, Lihong Chen ${ }^{3,}$ and Xinyu Chang ${ }^{4}$ \\ Institute of mechanics, Chinese Academy of Sciences, Beijing China, 100190
}

\begin{abstract}
A design methodology has been developed for the base flow of streamline-traced hypersonic inlet. Limited by the inlet length, the truncated Busemann flow results in a curved shock wave accompanied with large total pressure loss near the axis. ICFA is then utilized to maintain the shock shape as straight as possible, downstream which an internal flow defined by spline surface is optimized for maximum total pressure recovery. Though the shock wave still curves, it only occurs in a very small region close to the axis. Accordingly, compared to truncated Busemann flow with same length and contraction ratio, the developed base flow contributes to a higher total pressure.
\end{abstract}

\section{Nomenclature}

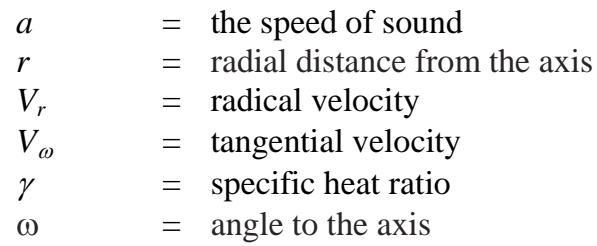

\section{Introduction}

$\mathrm{F}$ or best hypersonic airbreathing engine performance, the inlet is a critical component to provide a large amount of efficient compression. Design concepts for high performance scramjet inlets were well documented in the literature, which can be characterized in terms of the planar inlets and the inward-turning inlets. In planar inlets, the flow compressions are achieved by a series of planar or quasi-planar shock waves (ramp compression inlet ${ }^{[1]}$, sidewall compression inlet ${ }^{[2]}$ and $3 \mathrm{D}$ inlet ${ }^{[3]}$ ). Whereas, the inward turning inlets are designed to capture a portion of a known flowfield by specifying a leading edge and tracing the capture-perimeter streamlines through the baseline flowfield to create the inviscid waverider surfaces ${ }^{[4,5]}$. Designed for full mass capture, the inward turning inlets avoid additional drag due to the spillage at design condition.

The Busemann inlet has received considerable attention lately as a classical streamline-traced airbreathing inlet ${ }^{[6,7]}$. Whereas it has very high inviscid pressure recovery, even at off-design operation ${ }^{[8]}$, it is longer than the traditional outward turning inlet with the same capture area. Limited by the restriction of overall weight and size of the scramjet, it is always needed for an inlet designer to compromise between the size and the performance of an inlet. Therefore in the practical application, the inlet was generally shortened by truncating the isentropic compression surface ${ }^{[9-11]}$. Nevertheless, the flow pattern would be altered after truncation, especially near the axis. The coalescence of conical compression waves at the origin can not be realized and there is a curved shock wave that intersects with the axis at a point downstream the origin. The curvature of the shock wave would result in a higher total pressure loss near the axis, even a normal Mach disk. The flow then becomes non-uniform at the exit ${ }^{[12]}$.

Undoubtedly, the base flowfield has dominant effect on the inlet performance. The performance of truncated Busemann inlet would be degraded. The aim of this paper is to develop a better base flow to improve inlet performance.

\footnotetext{
${ }^{1}$ Associate Professor, Key Laboratory of High Temperature Gas Dynamics, yuelj@imech.ac.cn.

2 Assistant Professor, Key Laboratory of High Temperature Gas Dynamics.

${ }^{3}$ Associate Professor, Key Laboratory of High Temperature Gas Dynamics, Member AIAA.

${ }^{4}$ Professor, Key Laboratory of High Temperature Gas Dynamics, Member AIAA.
} 


\section{Truncated Busemann Flow}

The Busemann inlet should be shortened without severe deterioration in performance. Previous researches have analyzed the effect of the truncation angle on the base flowfield in order to determine the suitable truncated location. For incoming flow Mach number of 5.05 and exit Mach number of 3.0, a $5^{\circ}$ truncated inlet provides a better performance. Its length is about 2/3 that of the full Busemann inlet, whereas the inviscid total pressure recovery has been maintained up to $90.02 \%$. Further increase in the angle would contribute to a rapid increase in total pressure loss, while only a little decreases in the compression length ${ }^{[12]}$.

Fig. 1 shows the flow patterns for the $5^{\circ}$ truncated Busemann flow. By comparison, Fig. 2 illustrates the schematic of the full Busemann flow field. Without truncation, the axisymmetric internal flow consists of a set of conical Mach waves followed by a free-standing conical shock wave. All the conical Mach waves and the single freestanding conical shock wave coalesce at the apex "O”. The inlet takes the uniform parallel flow since the freestanding conical shock wave is canceled at the body shoulder. After truncation, the sharp leading edge corner results in the formation of an oblique shock wave. Moreover, the compression waves from the curved surface would not coalesce at the origin " 0 ", but intersect with the shock wave. A curved shock wave occurs that intersects with the axis at a point downstream the origin ' 0 '. The reflected conical shock wave also impinges at a location downstream the shoulder rather than the shoulder in the non-truncated case. Although the total pressure recovery is still high, the curvature of the shock wave results in the high total pressure loss near the axis, even a normal Mach disk. As shown in the Fig. 1, the total pressure recovery near the axis has come down to $65.7 \%$, rather than $97.2 \%$ that is far from the axis. This phenomenon decreases the total pressure; on the other hand, it degrades the flow uniformity. Correspondingly, if we can control the compression waves to maintain the shock strength, above disadvantages can be perhaps limited.

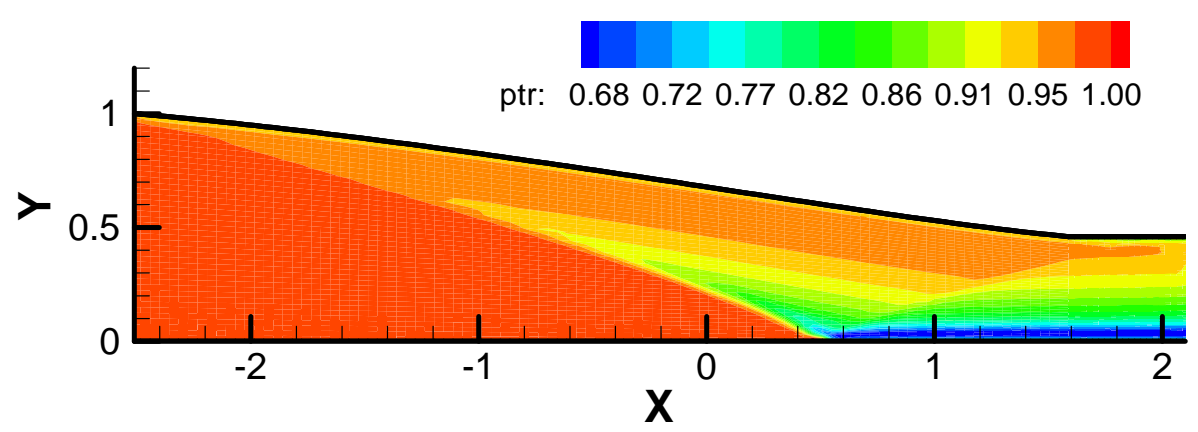

a) Total pressure contour

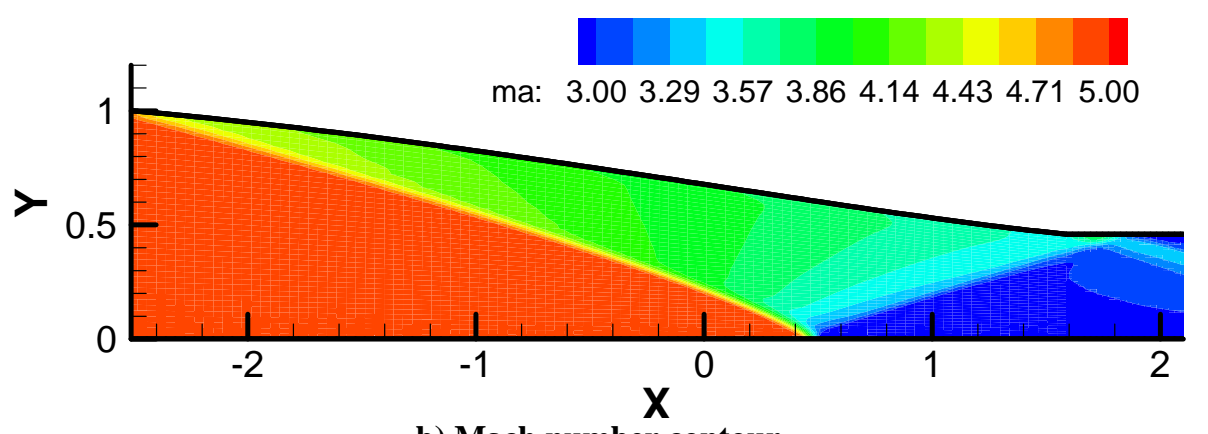

b) Mach number contour

Figure 1. Flow field of $5^{\circ}$ truncated Busemann flow (incoming flow Mach 5.05) 


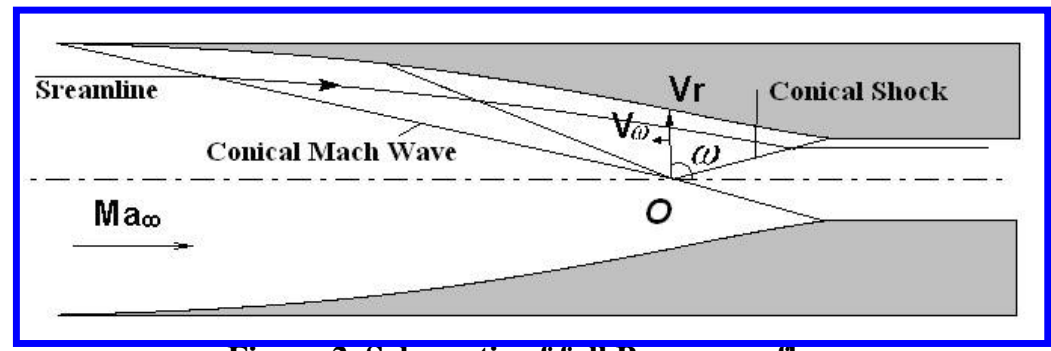

Figure 2. Schematic of full Busemann flow

\section{Internal Conical Flow}

In order to control the shock strength along radical direction, we had better maintain the shock wave as straight as possible to avoid the larger shock angle around the axis.

S. Molder has ever demonstrated four types of axisymmetric conical flow ${ }^{[13]}$. Except the external conical flow, Busemann flow, ICFA (Internal Conical Flow A) was analyzed as a convergent flow, in which parallel flow passes through a conical shock and converges towards the axis, as shown in Fig.3. This flow can also be described by the Taylor-Maccoll equation:

$$
V_{r}^{\prime \prime}=\frac{(\gamma-1)\left(2 V_{r}+V_{r}^{\prime} \operatorname{ctg} \omega\right)\left(1-V_{r}^{2}-V_{r}^{\prime 2}\right) / 2}{V_{\omega}^{2}-a^{2}}
$$

Where $V_{\mathrm{r}}$ is the velocity in the $\mathrm{r}$ direction, nondimensionalized with respect to the escape speed.

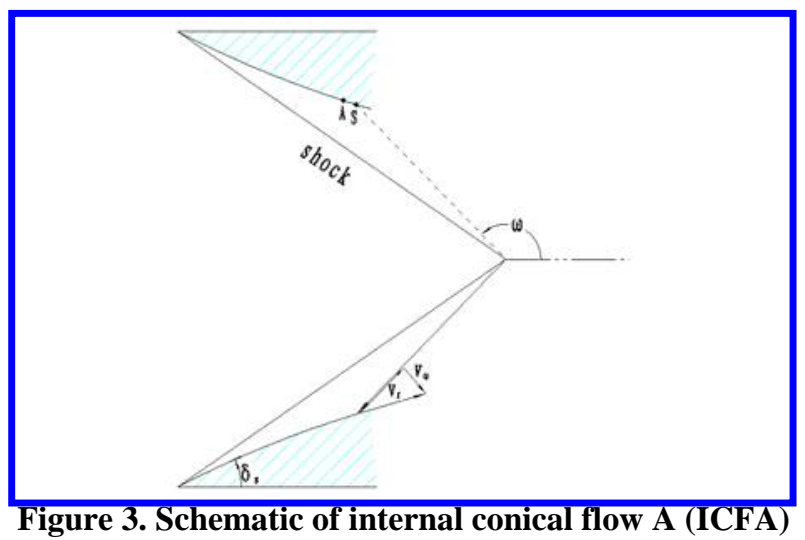

The ICFA flow starts from a straight internal conical shock which leads to constant total pressure recovery behind the shock, which will contribute to the flow uniformity of the total pressure recovery within the inlet. Unfortunately, from the equation, we see that there is so-called singularity condition when the denominator equals 0 . The numerical solutions shows that near the singularity $\mathrm{V}_{\omega}$ approaches the speed of sound, and consequently $\mathrm{V}_{\mathrm{r}}$, approaches infinity. It was noted also that near the singularity the surface starts turning extremely sharply further away from the freestream flow. The existence of the singularity indicates that ICFA can not be realized completely and regular shock reflection at the axis is impossible.

Nevertheless, we can expect to maintain the initial shock wave as straight as possible by use of ICFA. The singular ray is a characteristic line because the tangential velocity is the speed of sound. Therefore the shape of initial shock would be only influenced by the surface upstream the singularity. In order to obtain a nearly straight shock wave, the body surface of ICFA can be specified till a location " $\mathrm{A}$ " close to singularity point "S".

A curve is subsequently connected from point "A" to the throat, as shown in Fig.4. Though the shock pattern would be interfered by the surface downstream the connecting point "A", the effect would be limited within a small region close to the axis. 


\section{Optimization Method and Numerical Setup}

The connecting curve AD is also important for the base flow performance, which will influence the initial shock around the axis and the reflected shock wave. In this paper, optimization was utilized to search the best curve with maximum total pressure recovery.

In order to compare with the truncated Busemann flow, the length and contraction ratio of optimized base flow were specified to equal to those of the truncated Busemann flow. Meanwhile, the angle of shock emanating from the leading edge were designed as the same as the truncated Busemann inlet. The body contour is composed of the part of ICFA section and a successive NURBS that is determined by 4 vertices, such as the connecting vertex " $A$ ", the inlet shoulder "D", the optimization vertices "B" and " $C$ ", as illustrated in Fig.4. The coordinates of B, C were chosen to be the optimization variables, whose y-coordinates were bounded to be greater than that of the vertex $\mathrm{D}$ and less than the vertex A.

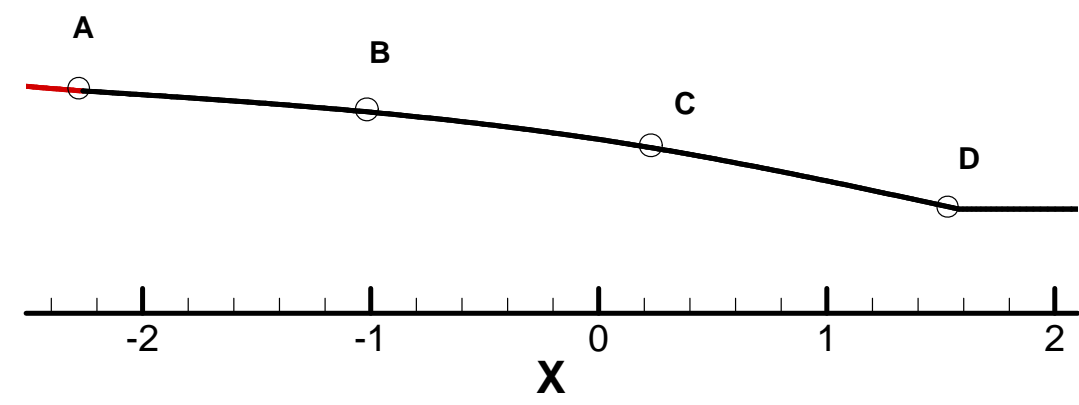

Figure 4. Schematic of body surface of the designed base flow

Generalized Pattern search (GPS) algorithm, whose convergence was proved by Torczon ${ }^{[14]}$, is an local optimization method suitable to solve the nonlinear unconstrained optimization problems, bound constrained, and linearly constrained optimization problems. It differs from the traditional optimization methods with the cancellation of gradient computation. The GPS algorithms class designed by Torczon unifies a wide class of useful derivativefree algorithms for unconstrained optimization. Lewis and Torczon extended the GPS framework to bound constrained optimization $^{[15]}$, and, more generally for problems with a finite number of linearly constraint. Audet and Dennis allow extended value functions ${ }^{[16]}$. Furthermore, Torczon analyzes a pattern search method for nonlinearly programming without derivatives based on filter methods for step acceptance.

The GPS algorithms include two steps: search steps and poll steps. The search steps are preferred to make a global exploration of the variable space, and they might use inexpensive surrogate objective and constraints to predict points that constitute improvements to the real problem. It is believed that this is crucial for the application of direct search methods to a large class of the engineering design problems because applying these methods directly to the actual problem would be in many cases prohibitively expensive.

The characteristics of base flow were studied by inviscid computations, which featured a second order upwind discretization scheme and the AUSM flux type. The incoming flow conditions were given and the exit flow was calculated by extrapolation. The computation was included in a subroutine of the GPS optimization code, which was passed the geometry parameters and was expected to return the total pressure recovery of the inlet. Maximum total pressure recovery was chosen to be the goal of the optimization, and $10^{-6}$ the convergence threshold.

\section{Design of the Base Flow}

\section{A. Shape determination}

The design methodology described in previous sections is applied to a hypersonic base flow. The free stream Mach number is assumed to be 5.05 , total contraction ratio is 4.78 . The ramp angle of the leading edge is chosen as $5^{\circ}$.

It was well known that the initial shock wave would be curved even in a straight internal conical flow. In order to help maintain the straight shock shape, the flow should be expanded downstream the leading edge to intersect the expansion fans with the shock wave. So the ICFA surface would be like a bell, as shown in Fig.5. On the other hand, the expansion process would decrease the compression efficiency because the hypersonic inlet is used to diffuse the air flow. Fortunately, the section is not so long, only 2/5 of the radius, as shown in Fig.5. Fig.6 further plots the 
Mach number variation with the $\mathrm{X}$ coordinate. It is revealed that ICFA flow only accelerates a little downstream the shock. The negative effect of the expansion can be limited.

By optimization, the surface geometry is obtained, as shown in Fig.7. A good base flow is obtained by ICFA surface and subsequent spline curve. If we optimize the base flow without ICFA, lots of points would be needed to realize the qualified shock due to smooth curvature variation, especially downstream the leading edge. The calculation would be numerous and expensive.

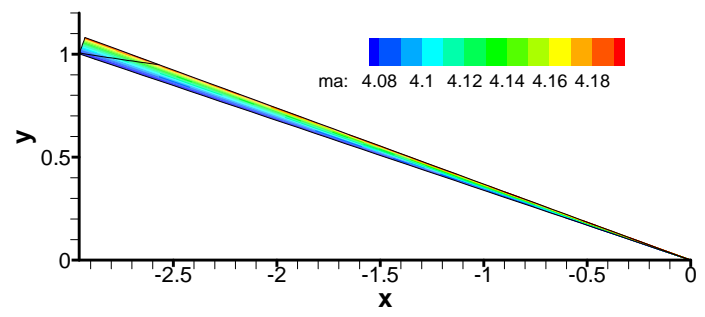

Figure 5. Flow and streamline of ICFA

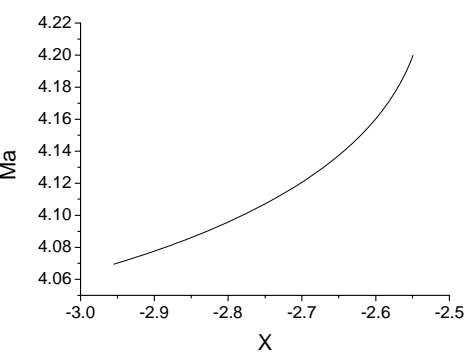

Figure 6. Mach number variation of ICFA

\section{B. Inviscid characteristics of the base flow}

To gauge the effectiveness of the designed base flow, its performance can be compared with the truncated Busemann flow. Their flow fields are exhibited in Fig.1 and Fig.7 respectively.

Fig.7(b) evidently reveals that the shock wave induced by the leading edge is approximately straight except in a small region close to the axis. It indicates that the ICFA surface behind the leading edge maintains the shock shape at best. Compared to Fig.1(a), only a little part of the shock is interfered by the compression surface, and the negative effect has been suppressed. The shock strength is controlled better than that in truncated Busemann flow. The total pressure can be therefore kept at a high level, as plotted in Fig.7(a). Note that the total pressure recovery near the axis is $76 \%$, much larger than $65.7 \%$ in truncated Busemann flow. Correspondingly, mass weighted averaged total pressure recovery reaches $94.94 \%$ for the designed base flow, $4 \%$ higher than the truncated Busemann flow.

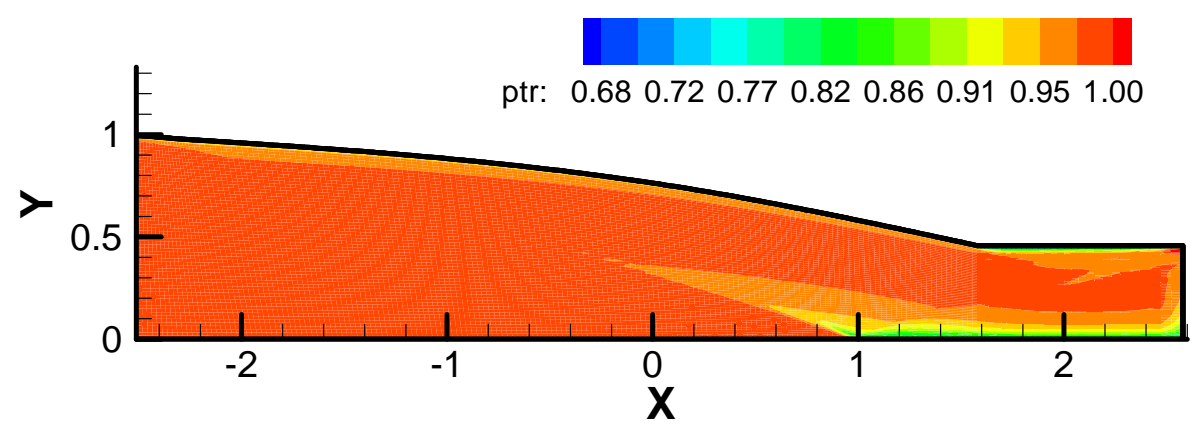

a) Total pressure contour

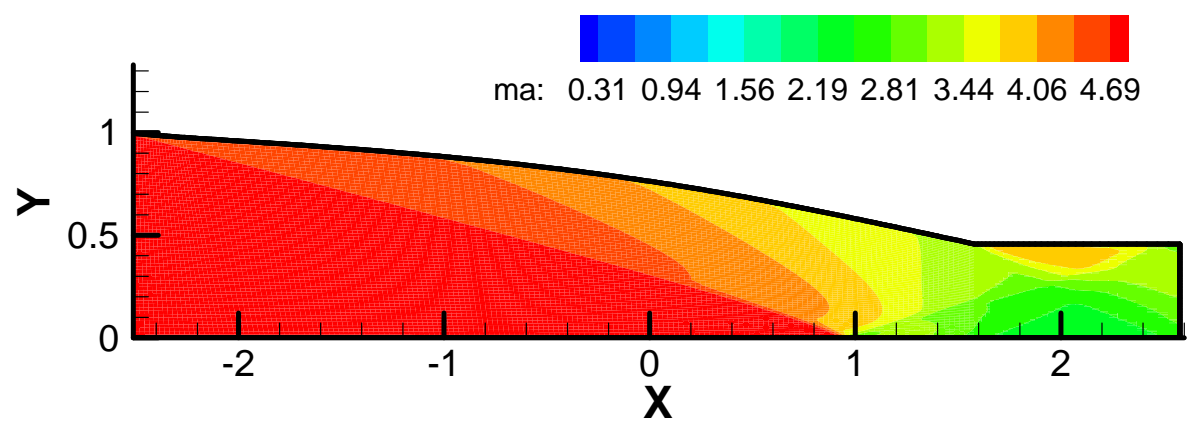

b) Mach number contour

Figure 7. Flow field of designed base flow (incoming flow Mach 5.05)

5

American Institute of Aeronautics and Astronautics 


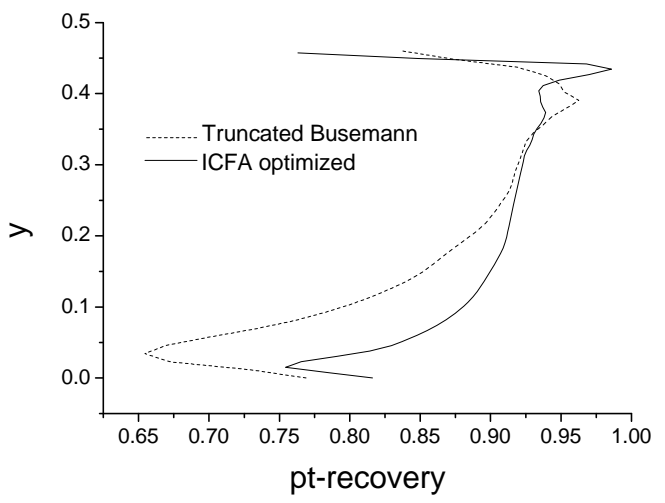

(a) Profile of total pressure recovery

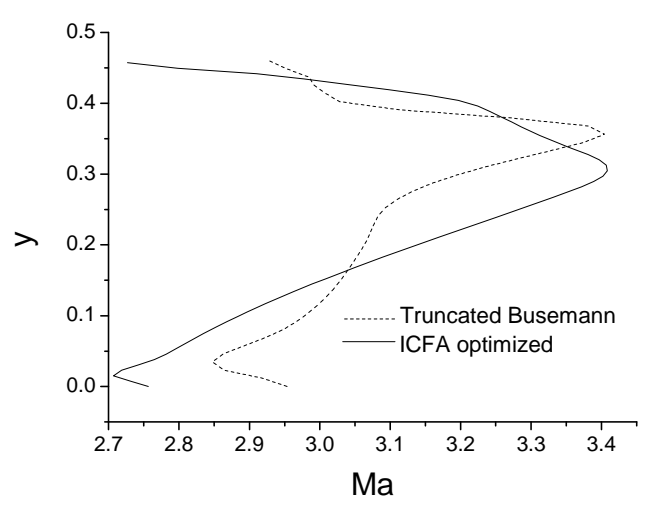

(c) Profile of Mach number

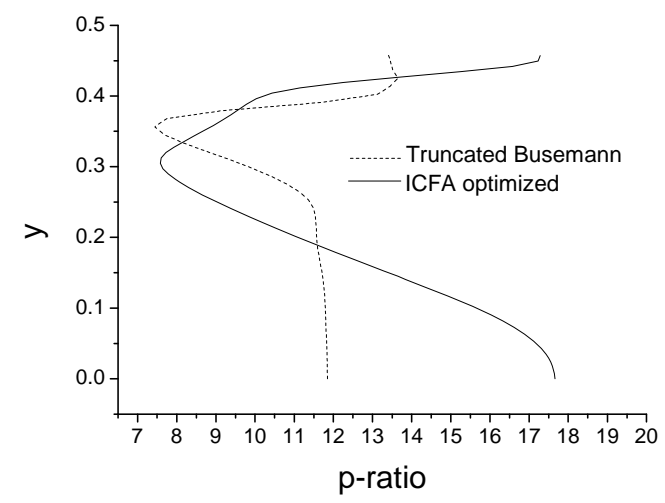

(b) Profile of static pressure ratio

\section{Figure 8. Flow parameters at the exit of the base flow}

Additionally, the reflected shock is also nearly a straight line in the designed base flow, and impinges on the location downstream of the shoulder. In fact, the shock is not canceled at the shoulder even in the truncated Busemann flow although the full Busemann flow can theoretically realize the uniform flow at the throat. Fig.8 depicts the profile of the flow parameters at the exit. It is noted that the total pressure is more uniform for the designed base flow than truncated Busemann flow because the shock wave emanating from the leading edge is much straighter. However, its uniformity of the static pressure ratio is worse, which can be attributed to the further downstream impingement location of the reflected shock relative to the shoulder. The farther is the distance of the impingement location from the shoulder, the worse uniformity does the flow.

Due to the straighter shock wave in the designed base flow, the intersection point of the shock with the axis is further downstream of that in truncated Busemann flow. Corresponding streamline traced inlet would be defined with its internal contraction ratio of 1.59, less than 1.68 of the truncated Busemann inlet.

\section{Conclusion}

A design methodology has been presented for the base flow of streamline-traced hypersonic inlet in order to overcome the disadvantage in truncated Busemann flow. Limited by the inlet length, the truncated Busemann flow results in a curved shock wave accompanied with large total pressure loss near the axis.

ICFA is utilized to maintain the shock shape as straight as possible. To overcome the inherent singularity problem, ICFA surface is specified till a location very close to the singular point. An optimized spline curve is subsequently connected for maximum exit total pressure.

The characteristic of base flow was investigated with incoming Mach number 5.05 and contraction ratio of 4.78. The ramp angle of the leading edge is chosen as $5^{\circ}$. Under the limitation of same length and contraction ratio, the truncated Busemann flow was also analyzed for comparison. Though the shock pattern is interfered by the compression surface downstream the connecting point, the effect has been limited within a small region close to the 
axis. The developed base flow therefore exhibits higher total pressure performance, $4 \%$ higher than truncated Busemann flow.

Although we have got a better base flow, it is possible to improve the optimization by increasing the number of controlling points on the spline curve.

\section{Acknowledgments}

The authors thank the assistance of Xiaojia Zhang in the numerical optimization. Current research program is sponsored and financially supported by the National Natural Science Foundation of China (Project No. 90716014).

\section{References}

${ }^{1}$ Matthew Brown, Neil R. Mudford, Andrew J. Neely and Tapabrata Ray, "Robust Design Optimization of Two-Dimensional Scramjet Inlets”, 14th AIAA/AHI Spaces and Hypersonic Systems and Technologies Conference, AIAA 2006-8140.

${ }^{2}$ Scott D. Holland, "Internal Aerodynamics of a Generic Three-Dimensional Hypersonic Scramjet Inlet at Mach 10", NASA TM-3476, 1995.

${ }^{3}$ Tetsuo Hiraiwa, Takeshi Kanda, Tohru Mitani, Yoshinari Enomoto, "Experiments on a Scramjet Engine with RampCompression Inlet at Mach 8 Condition”, 38th AIAA/ASME/SAE/ASEE Joint Propulsion Conference \& Exhibit, AIAA 2002-4129.

${ }^{4}$ F.S.Billig, Ajay P. Kothari, "Streamline Tracing: Technique for Designing Hypersonic Vehicles", Journal of Propulsion and Power, Vol.16, No.3, 2000, pp465-471.

${ }^{5}$ M. K. Smart, “Design of Three-Dimensional Hypersonic Inlets with Rectangular-to- Elliptical Shape Transition”, Journal of Propulsion and Power, Vol.15, No.3, 1999, pp408-416.

${ }^{6}$ Steven H. Walker, "Falcon Hypersonic Technology Overview", AIAA/CIRA 13th International Space Planes and Hypersonic Systems and Technologies Conference, AIAA 2005-3253.

${ }^{7}$ Mölder, S., “Busemann Inlet for Hypersonic Speeds”, Journal of Spacecraft and Rocket, Vol. 3, No. 8, 1966, pp1303, 1304.

${ }^{8}$ C.-J.Tam, R.A.Baurle, "Inviscid CFD Analysis of Streamline Traced Hypersonic Inlets at Off-Design Conditions", 39th AIAA Aerospace Sciences Meeting \& Exhibit, AIAA 2001-0675.

${ }^{9}$ F.S.Billig, "Supersonic Combustion Ramjet Missile”, Journal of Propulsion and Power, Vol.11, No.6, Nov.-Dec., 1995, P1139-1146.

${ }^{10}$ F.S.Billig, R.A.Baurle, C.-J.Tam, S.F.Wornom, "Design and Analysis of Streamline Traced Hypersonic Inlets”, 9th International Space Planes and Hypersonic Systems and Technologies Conference and 3rd Weakly Ionized Gases Workshop, AIAA 99-4974.

${ }^{11}$ F.S.Billig, L.S.Jacobsen, "Comparison of Planar and Axisymmetric Flowpaths for Hydrogen Fueled Space Access Vehicles”, AIAA/SAE/ASME/ASEE 39th Joint Propulsion Conference, AIAA 2003-4407.

${ }^{12}$ Yabin Xiao, Lianjie Yue, Peng Gong, and Xinyu Chang, "Investigation on a Truncated Streamline-Traced Hypersonic Busemann Inlet”, 15th AIAA International Space Planes and Hypersonic Systems and Technologies Conference, AIAA 20082634.

${ }^{13}$ Mölder, S., “Internal, axisymetrric, conical flow”, AIAA Journal, Vol. 5, No. 7, 1967, pp1252-1255.

${ }^{14}$ Torczon, Virginia, "On the Convergence of Pattern Search Algorithms", SIAM Journal on Optimization, Vol. 7, No. 1, 1997, pp1-25.

${ }^{15}$ Lewis, Robert Michael and Virginia Torczon, "Pattern Search Algorithms for Bound Constrained Minimization", SIAM Journal on Optimization, Vol. 9, No. 4, 1999, pp1082-1099.

${ }^{16}$ C. Audet and J.E. Dennis Jr., “Analysis of generalized pattern searches”, SIAM Journal on Optimization, Vol.13, No.3, 2003, pp889-903. 
This article has been cited by:

1. Samuel E. Otto, Charles J. Trefny, John W. Slater. 2016. Inward-Turning Streamline-Traced Inlet Design Method for LowBoom, Low-Drag Applications. Journal of Propulsion and Power 32:5, 1178-1189. [Abstract] [Full Text] [PDF] [PDF Plus]

2. Samuel E. Otto, Charles J. Trefny, John W. SlaterInward-Turning Streamline-Traced Supersonic Inlet Design Method for Low-Boom, Low-Drag Applications . [Citation] [PDF] [PDF Plus] 